\title{
A Case of Accommodative Insufficiency with Vergence Disorder in a 17-Year Old Student
}

\author{
Emmanuel Kanyika, Bright Vandyke Okyere* \\ Department of Optometry, Mzuzu University, Mzuzu, Malawi \\ Email address: \\ emmanuelkanyika2@gmail.com (E. Kanyika),brytokvandyke@yahoo.co.uk (B. V. Okyere) \\ ${ }^{*}$ Corresponding author
}

\section{To cite this article:}

Emmanuel Kanyika, Bright Vandyke Okyere. A Case of Accommodative Insufficiency with Vergence Disorder in a 17-Year Old Student. International Journal of Ophthalmology \& Visual Science. Vol. 5, No. 1, 2019, pp. 11-14. doi: 10.11648/j.ijovs.20200501.12

Received: November 22, 2019; Accepted: December 26, 2019; Published: January 7, 2020

\begin{abstract}
Accommodation Insufficiency (AI) is a condition in which a patient has an inability to focus or maintain focus at near. AI is mostly observed to occur with a vergence problem especially convergence insufficiency due to the close link between accommodation and convergence system of the eyes. Common symptoms seen in AI are diplopia, asthenopia and difficulty reading at near. Several management options are available including plus lenses for near work, optometric vision therapy and monitoring. A 17-year-old lady presented to the eye department with eyestrains, frontal headache, blurry vision at near with reading difficulties. According her, this problem started in 2018. Visual acuity (VA) for both distance and near was measured. Detailed anterior and posterior segments assessment revealed no anomalies, but binocular assessment showed reduced Amplitude of Accommodation (AOA), increased lag of accommodation, reduced Monocular Accommodative Facility (MAF) and Binocular Accommodative Facility (BAF), reduced Positive Relative Accommodation (PRA) and receded Near Point of Convergence (NPC). Based on the patient's complaint and what the assessment revealed, accommodative insufficiency with vergence disorder was made. Vision therapy and distance optical correction were prescribed to manage the problem. Accommodative Insufficiency can be treated effectively by an in-office/home-based program. The repetition of appropriate optometric vision therapy procedures at home will help resolve a patient's maladaptive visual condition over time.
\end{abstract}

Keywords: Accommodative Insufficiency, Accommodation, Binocular Vision, Convergence Insufficiency

\section{Introduction}

Accommodative insufficiency (AI) is an accommodative condition in which a person has difficulty stimulating accommodation to get a clear focus of targets of regard at near and it is characterised by lower Amplitude of Accommodation (AOA) than the person's age expected AOA and it is not associated with sclerotic changes of the crystalline lens due to aging, $[1,7,14]$. The age expected AOA is calculated using the Duane-Hofstetter's formula for minimum amplitude (15.0-0.25 x age) and can then be compared to the actual AOA of the patient. AOA of $2 \mathrm{D}$ or more below the age expected value, is abnormal [2-4]. Accommodative insufficiency is one of the commonest accommodative anomalies among children and patients with AI will struggle with schoolwork and may find it difficult reading because they experience eye fatigue and headaches after prolonged close work [5, 8]. Symptoms begin almost simultaneously with an increase in near work demand [15]. The inability to focus on near targets or to sustain clear vision for a period of time, diplopia, asthenopia, and difficulty reading with headache are the most frequent patient complaints [16]. It is caused by a number of factors such as alcohol, marijuana, medications (stimulants, phenothiazine, antihistamines, cycloplegics and others) and diseases such as malaria, anemia, mumps, measles, diabetes mellitus, multiple sclerosis, myotonic dystrophy [6].

To manage AI, the recommended sequential management starts with the correction of any refractive error present, added near lenses and visual therapy [2]. Literature has shown a high rate of comorbidity of AI and Convergence Insufficiency (CI) [2, 9]. CI is a binocular vision dysfunction characterized by the patient's inability to converge or sustain 
accurate convergence when focusing on near objects [10]. The inability to concentrate for long periods during near work can reduce the level of student achievement, so CI and AI are presented with negative factors in relation to health and quality of life as both interfere with reading and near work [10-13].

\section{Case Presentation}

On January 17 2019, a 17-year-old female secondary school student visited the eye department at Nkhata Bay district Hospital and complained of eyestrains, tearing, and blurry vision at near. She stated she was unable to read more than three pages because prints tend to be blurry and it was associated with frontal headache that is mostly felt after doing close work. She could not recall the exact date when the problem started but she stated that it started between August and September 2018. The symptoms were relieved when she stops reading. She had no remarkable ocular history. She was not on any medication and there was no significant family ocular and medical history.

\section{Ocular Examination}

\subsection{Visual Acuity}

The distance and near visual acuities were measured using Snellen chart in metric system and N-system card respectively.

Table 1. Visual Acuity measurements.

\begin{tabular}{ll}
\hline DISTANCE VISUAL ACUITY & \\
\hline Right Eye (RE) & $6 / 6$ \\
Left Eye (LE) & $6 / 6$ \\
NEAR VISUAL ACUITY & \\
Right Eye (RE) & $\mathrm{N} 8$ \\
Left Eye (LE) & $\mathrm{N} 8$ \\
Both Eyes (BE) & $\mathrm{N} 6$ (with difficulty) \\
\hline
\end{tabular}

\subsection{Anterior Segment Examination}

Table 2. Anterior segment findings.

\begin{tabular}{lll}
\hline Right eye (RE) & Structure & Left eye (LE) \\
\hline No abnormality detected & Eyelids/lashes & No abnormality detected \\
Clear, (-) injection, (-) growth & Conjunctiva & Clear, (-) injection, (-) growth \\
Clear, (-) stains with fluorescein & Cornea & Clear, \\
Clear, deep \& quiet & Anterior chamber & $(-)$ stains with fluorescein \\
Brown, flat \& pigmented & Iris & Clear, deep \& quiet \\
ERRL, (-) RAPD & Pupil & Brown, flat \& pigmented \\
Clear, (-) opacity & Lens & ERRL, (-) RAPD \\
Clear, (-) cells & Vitreous & clear, (-) opacity \\
\hline
\end{tabular}

\subsection{Posterior Segment Examination}

Table 3. Posterior segment findings.

\begin{tabular}{lll}
\hline Right eye & Structure & Left eye \\
\hline $\mathrm{CDR}=0.3$, ISNT rule followed, (-) peripapillary atrophy, & Optic disc & CDR=0.3, ISNT rule followed, (-) peripapillary atrophy, \\
Bright foveal reflex, (-) drusen, (-) oedema & Macula & Bright foveal reflex, (-) drusen, (-) oedema \\
No abnormality detected & Peripheral Retina & No abnormality detected \\
\hline
\end{tabular}

\subsection{Assessment of Binocular Vision}

The assessment comprised Ocular motility test, IPD, Refraction, AOA, phoria at near and distance, NPC, BI reserves, $\mathrm{BO}$ reserves, PRA, NRA, MAF, BAF and AC/A ratio.

Table 4. Binocular vision findings.

\begin{tabular}{ll}
\hline Cycloplegic refraction & RE: $+0.75 \mathrm{DS}$ \\
& LE: $+0.75 \mathrm{DS}$ \\
Subjective refraction & RE: $+0.50 \mathrm{DS} 6 / 6$ \\
IPD & LE: $+0.50 \mathrm{DS} 6 / 6$ \\
NPC & $60 \mathrm{~mm}$ \\
AOA (push up to blur) without & $20.5 \mathrm{~cm} / 28.6 \mathrm{~cm}$ \\
correction & RE: $4.20 \mathrm{D}$ \\
& LE: $4.40 \mathrm{D}$ \\
AOA with correction & BE: $6.45 \mathrm{D}$ \\
& RE: $3.75 \mathrm{D}$ \\
\hline
\end{tabular}

\begin{tabular}{ll}
\hline Distance phoria & Orthophoria \\
Near phoria & 8 XOP \\
AC/A Ratio (calc.) & $2.8: 1$ \\
Base-out near & $6 / 10 / 6$ \\
Base-in near & $8 / 15 / 10$ \\
NRA & +2.50 \\
PRA & -1.00 \\
MAF & RE:0cpm fails -2.00 \\
BAF & LE:0cpm fails -2.00 \\
MEM & $0 c p m$ fails -2.00 \\
\hline
\end{tabular}

There was no abnormality detected with eye movements in all gazes. Pain and double vision were not reported on moving the eyes in all gazes.

\section{Differential Diagnoses}

Convergence excess, Convergence insufficiency, Basic 
esophoria, Ill-sustained accommodation, Accommodative insufficiency, Accommodative infacility, Pseudo-convergence insufficiency and latent hyperopia.

\subsection{Diagnosis}

Accommodative insufficiency with vergence disorder (convergence insufficiency).

\subsection{Management Plan}

The patient and the guardian were educated on the nature of the condition and what could possibly be done to mitigate the problem.

A spectacle prescription of $+0.75 \mathrm{DS}$ for both eyes was prescribed to correct the refractive error.

Vision therapy was instituted;

Accommodative tromboning: use N8 sized target for fixation and move the target closer to and then away from patient. Ten cycles for 5 minutes thrice daily for month.

Pencil push ups were prescribed to the patient to be done for 10 minutes thrice a day for a month.

The patient was instructed to come for review on 17 February 2019.

\section{Second visit on $9^{\text {th }}$ April (REVIEW)}

The patient did not report on the set date because school was on recess but through the phone call the patient reported that there was an improvement after doing the vision exercises that were prescribed. So, the patient was encouraged to continue with the visual exercises and visit was rescheduled to $9^{\text {th }}$ April 2019.

On $9^{\text {th }}$ April 2019 the patient came for review and these are the findings which were measured on top of the prescription.

Table 5. Findings on review.

\begin{tabular}{ll}
\hline NPC & $\mathbf{5 c m} / \mathbf{8 c m}$ \\
\hline & RE:10D \\
AOA (push up to blur) & LE:10D \\
& BE:10.50D \\
Distance phoria & Orthophoria \\
Near phoria & $4 X O P$ \\
AC/A Ratio (calculated) & $4.4 / 1$ \\
Base-out near & $9 / 16 / 9$ \\
Base-in near & $8 / 15 / 10$ \\
NRA & $+2.50 \mathrm{D}$ \\
PRA & $-2.00 \mathrm{D}$ \\
MAF & RE: $6 \mathrm{cpm}$ \\
BAF & LE: $5 \mathrm{cpm}$ \\
MEM & $8 \mathrm{cpm}$ \\
\hline
\end{tabular}

\section{Discussion}

First visit assessment of the patient revealed that the AOA was less than the age expected. The minimum AOA for her age using the Hofstetter's formula (15.0-0.25xAge) is 10.75DS, but her accommodative amplitude was 6.45DS. Furthermore, (NPC) was receded, low AC/A ratio (calc.), MEM showed a lag in accommodation of $+1.25 \mathrm{D}$, NRA was normal while PRA was - 1.00 which was less than expected value. Facility testing revealed patient failing with -2.00 for both MAF and BAF.

A look at the accommodative data strongly indicates that all tests that findings of test that stimulate accommodation were less than expected leading to the diagnosis of accommodative insufficiency with vergence disorder. This was because of the linkage of accommodation and vergence systems (Near Triad). Less accommodation will mean less convergence exerted hence that's why the patient demonstrated an exophoria at near and the $\mathrm{AC} / \mathrm{A}$ ratio supports this.

One of diagnosis that could have been arrived at instead of accommodative insufficiency was Pseudo-convergence insufficiency. This was ruled out when we measured NPC with correction in place there was no improvement in NPC. Actually, in pseudo-convergence insufficiency all accommodative parameters are abnormal but when you correct the patient with spectacles the vergence problem (NPC) is solved leaving out accommodative parameters still below the expected.

Finally, the correction was only provided for distance vision and an ADD was not given to relieve symptoms because it could worsen the phoria at near. So visual therapy was instigated and after two months the patient reported symptomless.

\section{Conclusion}

In Accommodative Insufficiency, there is difficulty in stimulating accommodation to get a clear focus of objects at near. It presents with symptoms such as blurry vision at near, diplopia, headaches etc. These symptoms can be relieved by tests to stimulate the accommodative responses without resultant fatigue. Some of these tests include using minus lenses when the person is myopic and/ or accommodative therapy. Accommodative therapy increases the amplitude, accuracy, speed and ease of accommodative response.

\section{References}

[1] J. S. Cooper, et al, Care of the Patient with Accommodative and vergence Dysfunction, Lindbergh Blvd: American Optometric Association, 2011.

[2] M. Scheiman and B. Wick, Clinical Management of Binocular Vision: Heterophoric, Accommodative and Eye Movement Disorders. 4th. New York: Wolters Kluwer, 2015. pp. 335-365.

[3] Duam KM. Accommodative Dysfunction. Doc Ophthalmol 1983; 55: 177-98.

[4] Hofstetter HW. Useful age-amplitude formula. Optom World 1950; 38: 42-5.

[5] Eanes TH. "Low fusional convergence as a factor in reading disability”. American Journal of Ophthalomology 17.8 (1934): 709-710.

[6] W. J. Benjamin, etal Borish's Clinical Refraction. 2nd. Philadelphia: Butter-Heinmann, 2006. pp. 114-115.

[7] Duke-Elder S. The practice of refraction, 5th ed. St. Louis: CV Mosby, 1987: 431-59. 
[8] Borsting E, Rouse MW, Deland PN, et al. Association of symptoms and convergence and accommodation in school-age children. Optometry. 2003; 74 (1): 25-34.

[9] Marran LF, De Land PN, Nguyen AL. Accommodative insufficiency is the primary source of symptoms in children diagnosed with convergence insufficiency. Optom Vis Sci. 2006; 83 (5); 281-9.

[10] Copper J, Jamal N. Comvergence insufficiency: a major review. Optometry. 2012; 83 (4): 137-58.

[11] Shin HS, Park SC, Parh CM. Relationship between accommodative and vergence dysfunctions and academic achievement for primary school children. Ophthalmic Physiol Opt. 2009; 29 (6): 615-24.
[12] Borsting E, Mitchell GL, Kulp MT, et al. Improvement in academic behaviors following successful treatment of Convergence insufficiency. Optom Vis Sci. 2012; 89 (1): 12-8.

[13] Clinical Management of Binocular Vision: Heterophoric, Accommodative, and Eye Movement Disorders; Scheiman M, Wick B (Eds); Lippincott Williams \& Wilkins, 2002.

[14] AOA Optometric Clinical Practice Guideline; Care of the Patient with Accommodative and Vergence Dysfunction, 1998.

[15] Daum KM. Accommodative Insufficiency. Am J Optom Physiol Opt 1983; 60 (5): 352-359.

[16] Sterner B, Gellerstedt M, Sjo A. Accommodation and the relationship to subjective symptoms with near work for young school children. Ophthal Physiol Opt. 200626 (2): 148-155. 